\title{
Chylous Ascites: A Rare Initial Presentation of Pancreatic Carcinoma
}

\author{
Ammarah Ghafoor ${ }^{1}$, Junaid Zia Hashmi ${ }^{2}$, Abdul Mateen ${ }^{3}$ and Qurat ul Ain Hashmi ${ }^{3}$ \\ ${ }^{1}$ Department of Internal Medicine, Nishtar Hospital, Multan, Pakistan \\ ${ }^{2}$ Department of General Surgery, Nishtar Hospital, Multan, Pakistan \\ ${ }^{3}$ Department of Radiation Oncology, Multan Institute of Nuclear Medicine and Radiology, Multan, Pakistan
}

\begin{abstract}
Chylous ascites as first manifestation of pancreatic carcinoma is very rare. Usual presentations of pancreatic malignancy are abdominal discomfort, back pain, obstructive jaundice and duodenal obstruction. Development of chylous ascites during the course of the disease signifies poor prognosis. Malignancy and tuberculosis are the most common causes of this condition in developed and developing countries, respectively. Diagnosis is made on elevated triglyceride levels in ascitic fluid examination. Management is directed towards the treatment of primary cause and the chylous ascites. High protein and medium chain triglyceride diet is usually advised along with Orlistat for managing this condition. Peritoneovenus shunting can also be done but at the risk of complications. Our patient developed chylous ascites early in the course of the pancreatic carcinoma. CT scan abdomen was non-conclusive and cervical lymph node biopsy later on revealed that the primary disease was occult pancreatic carcinoma.
\end{abstract}

Key Word: Chylous ascites, Triglyceride level, Pancreatic carcinoma.

How to cite this article: Ghafoor A, Hashmi JZ, Mateen A, Hashmi QUA. Chylous Ascites: A Rare Initial Presentation of Pancreatic Carcinoma. J Coll Physicians Surg Pak 2020; 30(09):989-991.

\section{INTRODUCTION}

Pancreatic carcinoma rarely presents initially with chylous ascites, which is an uncommon condition. Chylous ascites is rather associated with the treatment of malignancy in the form of advanced and minimally invasive techniques. ${ }^{1}$ Chylousascites occurs only once in 20,000 cases of ascites; and tuberculosis is supposed to be the major cause in developing countries. ${ }^{2}$ Other causes are divided into two categories: traumatic and non-traumatic. Non-traumatic etiologies are infectious, cirrhotic, cardiogenic and neoplastic diseases. In developed countries, malignancy and cirrhosis cause $2 / 3^{\text {rd }}$ of these cases. Despite better and advanced treatment options, it is associated with very high mortality of $40-70 \% .^{3}$

It is characterised by milky white appearance of the fluid. Among all parameters, the level of triglycerides is the most important one in the diagnosis of this condition. A level of more than $187 \mathrm{mg} / \mathrm{dl}$ in the ascitic fluid is diagnostic of chylous ascites. $^{4}$

Correspondence to: Dr. Junaid Zia Hashmi, Ward No. 5, Surgical Unit IV, Nishtar Hospital, Nishtar Road, Multan, Pakistan

E-mail: juneehashmi@yahoo.com

Received: May 15, 2019; Revised: September 02, 2019;

Accepted: October 22, 2019

DOI: https://doi.org/10.29271/jcpsp.2020.09.989
Markers such as CA19-9, CA 125, adenosine deaminase and others are useful in diagnosing the etiology. Here, we reporta case of chylous ascites which was associated with occult pancreatic cancer with enlarged bilateral cervical lymph nodes.

\section{CASE REPORT}

A 20-year male patient, resident of Mianwali, Punjab presented in outpatient department (OPD) of MINAR in November 2018 with complaint of abdominal distention for six months and pain abdomen for three months. It was associated with weight loss, dry cough, and shortness of breath. There was no history of fever. There was no any past surgical history, but the patient had multiple visits to general physician for weight loss and abdominal distention. His mother was diabetic and father died of chronic kidney disease. No other significant family history of any disease was given.

On examination, the patient was a young male of lean body mass and grossly distended abdomen. His vitals were in normal ranges and he was afebrile. Pallor was positive, right-sided cervical lymph nodes were palpable and mobile with firm consistency. Abdomen was grossly distended with central umbilicus and intact hernia orifices. Fluid thrill and shifting dullness were positive. Bowel sounds were audible. Central nervous system, respiratory system and gastrointestinal examination was unremarkable.

His hemoglobin was $15.8 \mathrm{~g} / \mathrm{dl}$ and total leukocyte count (TLC) was $17,300 / \mathrm{mm}^{3}$. CEA was $13.79 \mathrm{ng} / \mathrm{ml}$, CA $19-9$ was $436.7 \mathrm{U} / \mathrm{ml}$, 
and lactate dehydrogenase (LDH) was $243 \mathrm{U} / \mathrm{L}$. Renal parameters, liver function tests and electrolytes were normal. CT abdomen showed moderate to severe ascites with omental thickening/caking, mesenteric and epigastric lymphadenopathy (Figure 1). Ascitic fluid was milky white unappearance (Figure 2) and biochemical examination showed triglyceride level of $544 \mathrm{mg} / \mathrm{dl}$. Cervical lymph node biopsy revealed metastatic adenocarcinoma of pancreatic origin. Immunohistochemistry stains were positive for cytokeratin 7, cytokeratin 19 and CDX-2. Raised CA19-9 and appearance of CK7, CK 19 and CDX-2 indicated the presence of an occult pancreatic carcinoma.

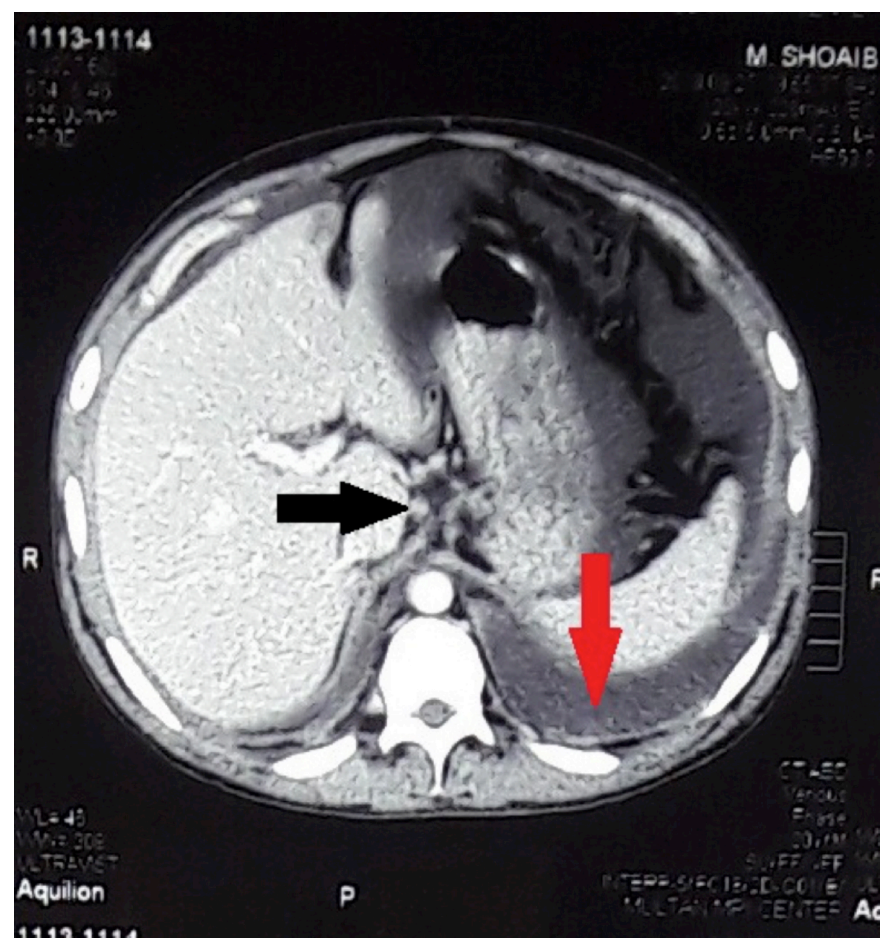

Figure 1: CT scan abdomen showing moderate ascites (red arrow) and epigastric lymphadenopathy (black arrow).

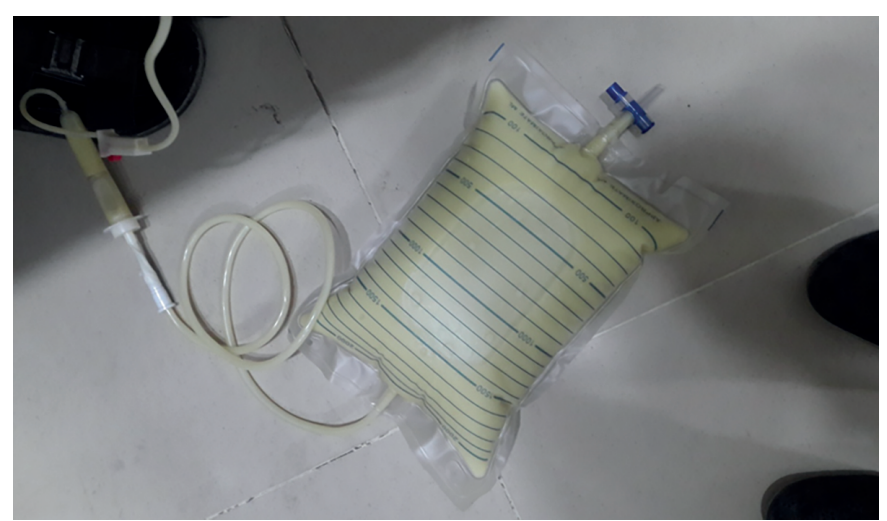

Figure 2: Milky white turbid fluid drained on peritoneal paracentasis.

Patient was advised medium chain triglycerides, high protein diet and orlistat for managing chylous ascites. The patient was put on the regime of cisplatin and gemcitabin. He was given 6 cycles of chemotherapy over a course of two months as a palliative therapy for advanced occult pancreatic carcinoma.

\section{DISCUSSION}

Pancreatic carcinoma presents according to the site of the malignancy. Malignancy of the head of pancreas presents with obstructive jaundice, duodenal obstruction and that of body and tail with vague signs and symptoms such as abdominal discomfort, back pain and rarely as chylous ascites. Chylous ascites usually presents with abdominal distention and pain. In developed countries, malignancy and portal hypertension are the major causes of chylous ascites among adult population. ${ }^{5}$ In our patient, occult pancreatic cancer was the cause of this condition, which was diagnosed on cervical lymph node biopsy. Chylous ascites associated with malignancy carries poor prognosis. ${ }^{6}$

Ascitic fluid examination after paracentesis is most helpful in diagnosing the chylous origin of peritoneal fluid. On physical examination, chyle is a milky, turbid fluid. Further microscopic examination of cell count, glucose, total protein, albumin, amylase, LDH and triglyceride level are also required. Information about gram staining and culture is usually a guide about the etiology. Triglyceride level of more than $187 \mathrm{mg} / \mathrm{dl}$ is diagnostic of chyle. ${ }^{4}$ CT scan, cytological examination and peritoneal biopsy after laparoscopy are also required to establish the cause. In our patient, the fluid was turbid. Total proteins were $8 \mathrm{~g} / \mathrm{dl}$ and triglyceride level was $544 \mathrm{mg} / \mathrm{dl}$.

Lymphoscintigraphy and lymphangiography are the newer modalities that help in patient selection for surgical intervention. These studies show abnormal lymph nodes and sites of leakage from lymphatic channels. These studies require special equipmentand qualified operators, which make these investigations expensive and unfavourable most of the time. ${ }^{7}$ These investigations were not carried out in our patient.

Management of this condition includes treating the ascites and the cause at the same time. Dietary restriction of long chain fatty acids and increase in protein diet are the mainstay of management. Patients are advised to take medium chain triglycerides because of their direct absorption through intestines, thus decreasing the flow of chyle. ${ }^{8}$ Orlistat is the drug of choice as it decreases the breakdown of triglycerides.

Our patient had stage IV occult pancreatic carcinoma as the primary cause of chylous ascites, so he was given six cycles of chemotherapy to treat the primary pathology. He was advised diet containing medium chain triglycerides, high protein and also orlistat to manage the chylous ascites. Progression of the disease process slowed down only slightly by these manoeuvers. Patient got mild symptomatic relief due to a mild decrease in abdominal distention.

LeVeen and Denver shunts are used for peritoneovenous shunting of chylous fluid. Although these shunts help in maintaining better hemodynamics and immunological status, they are associated with increased rates of complications. ${ }^{9}$ Shunting was not done in our patient, external drainage of ascites was carried outand patient was put on palliative chemotherapy. 
In summary, this case underscores the need for keeping pancreatic malignancy in the differential diagnosis of the diseases causing chylous ascites.

\section{PATIENT'S CONSENT:}

Informed consent was taken from the patient to publish this case.

\section{CONFLICT OF INTEREST:}

There is no conflict of interest in publishing of this case report.

\section{AUTHORS' CONTRIBUTION:}

AG: Abstract, literature search and discussion.

$\mathrm{JZH}$ : Introduction and discussion.

AM, QUAH: Literature search and discussion.

\section{REFERENCES}

1. Cárdenas A, Chopra S.Chylous ascites. Am J Gastroenterol 2002; 97(8):1896-900.

2. Browse NL, Wilson NM, Russo F, Al-HassanH, Allen D.R. Aetiology and treatment ofchylous ascites. Br J Surg 1992; 79(11):1145-5.
3. Aalami OO, Allen DB, Organ CH Jr. Chylous ascites: A collective review. Surgery 2000; 128(5):761-78.

4. Thaler MA, Bietenbeck A, Schulz C, Luppa PB. Establishment of triglyceride cut-off values to detectchy-lous ascites and pleural effusions. Clin Biochem 2017; 50(3):134-8.

5. Bhardwaj R, Vaziri H, Gautam A, Ballesteros E, Karimeddini D, Wu GY. Chylous ascites: A review of pathogenesis, diagnosis and treatment. J Clin Transl Hepatol 2017; 6(1): 105-113.

6. Mandavdhare HS, Sharma V, Singh H, Dutta U. Underlying etiology determines the outcome inatraumatic chylous ascites. Intractable Rare Dis Res 2018; 7(3):177-81.

7. Al-Busafi SA, Ghali $P$, Deschênes $M$, Wong P. Chylous ascites: Evaluation and management. ISRNHepatol 2014; 2014:240473.

8. Almakdisi T, Massoud S, Makdisi G. Lymphomas and chylous ascites: Review of the literature.Oncologist 2005; 10(8):632-5.

9. Makino $Y$, Shimanuki $Y$, Fujiwara N, Morio $Y$, Sato $K$, Yoshimoto J, et al. Peritoneovenous shunting forintractable chylous ascites complicated with lymphangioleiomyomatosis. Intern Med2008; 47(4):281-5. 